\title{
Dynamical evolution of Oort cloud comets to near-Earth space
}

\author{
Olga A. Mazeeva $\dagger$ \\ Department of Computational and Celestial Mechanics, South Ural State University, \\ Chelyabinsk, 454080, Russia \\ email: omega@susu.ac.ru
}

\begin{abstract}
The dynamical evolution of $2 \cdot 10^{5}$ hypothetical Oort cloud comets by the action of planetary, galactic and stellar perturbations during $2 \cdot 10^{9}$ years is studied numerically. The evolution of comet orbits from the outer $\left(10^{4} \mathrm{AU}<a<5 \cdot 10^{4} \mathrm{AU}, a\right.$ is semimajor axes $)$ and the inner Oort cloud $\left(5 \cdot 10^{3} \mathrm{AU}<a<10^{4} \mathrm{AU}\right)$ to near-Earth space is investigated separately. The distribution of the perihelion $(q)$ passage frequency in the planetary region is obtained calculating the numbers of comets in every interval of $\Delta q$ per year. The flux of long-period (LP) comets (orbital periods $P>200 \mathrm{yr}$ ) with perihelion distances $q<1.5$ AU brighter than visual absolute magnitude $H_{10}=7$ is $\sim 1.5$ comets per year, and $\sim 18$ comets with $H_{10}<10.9$. The ratio of all LP comets with $q<1.5$ AU to 'new' comets is $\sim 5$. The frequency of passages of $\mathrm{LP}$ comets from the inner Oort cloud through region $q<1.5 \mathrm{AU}$ is $\sim 3.5 \cdot 10^{-13} \mathrm{yr}^{-1}$, that is roughly one order of magnitude less than frequency of passages of LP comets from the outer cloud $\left(\sim 5.28 \cdot 10^{-12} \mathrm{yr}^{-1}\right)$. We show that the flux of 'new' comets with $15<q<31 \mathrm{AU}$ is higher than with $q<15 \mathrm{AU}$, by a factor $\sim 1.7$ for comets from the outer Oort cloud and, by a factor $\sim 7$ for comets from the inner cloud. The perihelia of comets from the outer cloud previously passed through the planetary region are predominated in the Saturn-Uranus region. The majority of inner cloud comets come in the outer solar system $(q>15 \mathrm{AU})$, and a small fraction $(\sim 0.01)$ of them can reach orbits with $q<1.5 \mathrm{AU}$. The frequency of transfer of comets from the inner cloud $\left(a<10^{4} \mathrm{AU}\right)$ to the outer Oort cloud $\left(a>10^{4} \mathrm{AU}\right)$, from where they are injected to the region $q<1.5 \mathrm{AU}$, is $\sim 6 \cdot 10^{-14} \mathrm{yr}^{-1}$.
\end{abstract}

Keywords. Oort Cloud, comets: general, solar system: general.

\section{Introduction}

A constant near-parabolic flux of comets (Marsden \& Williams 2005) originating from the Oort cloud (Oort 1950) is observed near the Earth orbit. The distribution of original reciprocal semimajor axes $(\varepsilon=1 / a)$ of observed comets shows a narrow peak in the range less than $10^{-4} \mathrm{AU}^{-1}$. Comets with $\varepsilon<10^{-4} \mathrm{AU}^{-1}$ are dynamically 'new' comets, i.e. presumably on their first passage in the planetary region. The flux of Earth-crossing $(q<1 \mathrm{AU})$ comets with $a>10^{4} \mathrm{AU}$ and absolute magnitudes $H_{10}<7$ is 0.2 per year (Bailey \& Stagg 1988). Oort (1950) argued that stellar perturbations are responsible for bringing of comets from the solar system cloud into the inner planetary region. Byl (1986) and Heisler \& Tremaine (1986) pointed out that the galactic tidal force is a more efficient mechanism of changing the perihelion distances of Oort cloud comets.

Hills (1981) proposed that a massive, unobserved inner cloud of comets with semimajor axes $a<10^{4} \mathrm{AU}$ exist. Comets of the inner cloud are weakly affected by external perturbations, except in the cases of passing of stars through the inner cloud. Duncan et al. (1987) simulated the formation of the Oort cloud and studied its subsequent evolution over an interval $4.5 \cdot 10^{9} \mathrm{yr}$, assuming that comets formed in the outer planetary region. The resulting spatial density of Oort cloud comets between $3 \cdot 10^{3} \mathrm{AU}$ and

$\dagger$ Present address: South Ural State University, 76 Lenina, Chelyabinsk, Russia. 
$5 \cdot 10^{4} \mathrm{AU}$ is $\sim r^{-3.5}$ and roughly corresponds to a distribution of semimajor axes $\sim a^{-1.5}$ (Duncan et al. 1987). This implies that $70 \%$ of comets have $a<10^{4}$ AU. Dones et al. (2000) found that the populations of the inner and outer clouds are roughly equal.

Comets which enter into the inner solar system are perturbed by the giant planets. The orbital energy $(\varepsilon=1 / a)$ of comets change at every perihelion passage. This process may be viewed as a slow diffusion of $1 / a$. The typical energy change $\Delta \varepsilon$ depends from the perihelion distance: $\Delta \varepsilon \approx 10^{-3} \mathrm{AU}^{-1}$ for $q<6 \mathrm{AU}, \Delta \varepsilon \approx 10^{-4} \mathrm{AU}^{-1}$ for $q \approx 10 \mathrm{AU}$ and $\Delta \varepsilon \approx 10^{-5} \mathrm{AU}^{-1}$ for $q \approx 20 \mathrm{AU}$ (Fernández 1981), and depends less significantly from the inclination. In the region $q<10$ AU the typical change in energy per perihelion passage due to planetary perturbations is greater than the initial energy of a comet. The region $q<10 \mathrm{AU}$ is called the 'loss cone' (Hills 1981).

The aim of this work is to study the dynamical evolution of comets from both the inner and outer Oort clouds to near-Earth space, to estimate the flux of 'new' and long-period comets for all perihelion distances in the planetary region.

\section{Model}

The dynamical evolution of $10^{5}$ comets of the outer Oort cloud (OOC) and $10^{5}$ comets of the inner Oort cloud (IOC), affected by planetary, galactic and stellar perturbations during $2 \cdot 10^{9} \mathrm{yr}$, is considered. The evolution of cometary orbits of the outer and inner Oort cloud is investigated separately. The initial semimajor axes $(a)$ of cometary orbits of the outer Oort cloud are distributed according to $a^{-1.5}$ in the range $(1-5) \cdot 10^{4} \mathrm{AU}$ (Duncan et al. 1987). Our model of the inner Oort cloud includes cometary orbits with semimajor axes distributed according to $a^{-1.5}$ in the interval $(0.5-1) \cdot 10^{4} \mathrm{AU}$.

The number of inner and outer cloud comets with perihelia in the range $(q, q+d q)$ is $N(q) d q$, where $N(q) \sim 1-q / a, q<a$ (Hills 1981). The initial inclinations $i$ are uniformly distributed in $\cos i$. For each initial orbit the argument of perihelion and the longitude of the ascending node are chosen randomly in the interval $\left(0^{\circ}, 360^{\circ}\right)$.

The perturbations of the four outer planets and the action of Galaxy are taken into account (Emel'yanenko 1999). Stellar perturbations are described by the impulse approximation (Ogorodnikov 1965). The distribution of parameters of stars and the frequency of their passages near the solar system are taken from Heisler et al. (1987).

The orbital elements are printed for each comet passage through the planetary region $\left(q<35 \mathrm{AU}\right.$ ) with $a>34.2 \mathrm{AU}$. The flux of comets with $\tau<10^{9} \mathrm{yr}$ and $10^{9}<\tau$ (yr) $<2 \cdot 10^{9}$ is investigated separately ( $\tau$ is the time of perihelion passage).

\section{Comparison with observations}

The comparison was made for observed and hypothetical comets with $a>8 \cdot 10^{3} \mathrm{AU}$ and $q<3$ AU. From the Catalogue of Cometary Orbits (Marsden \& Williams 2005) were taken the elements of orbits with quality classes 1 and 2, according to the classification proposed by Marsden et al. (1978). This excluded comets for which non-gravitational parameters have been determined. The observed and modeled $\varepsilon=10^{4} / a$ ( $a$ is the original semimajor axis) distributions are shown in Table 1.

The flux of comets with $T<10^{9} \mathrm{yr}$ in the intervals $\varepsilon(0.5 ; 0.75) \mathrm{AU}^{-1}$ and $\varepsilon(0.75 ; 1.0)$ $\mathrm{AU}^{-1}$ is approximately 1.5 times as large as the observed flux. The computed flux of comets in the range $\varepsilon(1.0 ; 1.25) \mathrm{AU}^{-1}$ (mainly comets coming from the inner cloud) is 8 times larger than the observed flux. The flux of comets with $10^{9}<T$ (yr) $<2 \cdot 10^{9}$ agrees better with the observations than that with $T<10^{9}$ yr (Table 1 ). 
Table 1. The $\varepsilon=10^{4} / a$ ( $a$ is the original semimajor axis) distributions of observed (Marsden \& Williams 2005) and hypothetical comets with $q<3$ AU. The distributions were normalized so that the number of comes with $\varepsilon(0.25 ; 0.5) \mathrm{AU}^{-1}\left(2 \cdot 10^{4}<a(\mathrm{AU})<4 \cdot 10^{4}\right)$ is unity. The flux of comets from the OOC and the combined flux of comets from the OOC and IOC for $T<10^{9}$ yr and $10^{9}<T$ yr $<2 \cdot 10^{9}$ is presented separately, where $T$ is the time of aphelion passage.

\begin{tabular}{|c|c|c|c|c|c|}
\hline \multirow{2}{*}{$\begin{array}{l}\varepsilon=10^{4} / a \\
\left(A U^{-1}\right)\end{array}$} & \multirow{2}{*}{$\begin{array}{c}\text { Observed } \\
\text { comets }\end{array}$} & \multicolumn{2}{|l|}{$T<10^{9} \mathrm{yr}$} & \multicolumn{2}{|c|}{$10^{9}<T(\mathrm{yr})<2 \cdot 10^{9}$} \\
\hline & & $\begin{array}{l}\text { OOC } \\
\text { comets }\end{array}$ & $\begin{array}{c}\text { (OOC+IOC) } \\
\text { comets }\end{array}$ & $\begin{array}{l}\text { OOC } \\
\text { comets }\end{array}$ & $\begin{array}{c}\text { (OOC+IOC) } \\
\text { comets }\end{array}$ \\
\hline $0.00-0.25$ & 0.714 & 0.994 & 0.741 & 0.969 & 0.878 \\
\hline $0.25-0.50$ & 1.000 & 1.000 & 1.000 & 1.000 & 1.000 \\
\hline $0.50-0.75$ & 0.250 & 0.404 & 0.348 & 0.217 & 0.236 \\
\hline $0.75-1.00$ & 0.179 & 0.258 & 0.243 & 0.074 & 0.135 \\
\hline $1.00-1.25$ & 0.036 & 0.050 & 0.282 & 0.039 & 0.071 \\
\hline
\end{tabular}

We then considered the distributions of original semimajor axes between $10^{4} \mathrm{AU}$ and $6 \cdot 10^{4} \mathrm{AU}$, in intervals of $5 \cdot 10^{3} \mathrm{AU}$, of the observed and hypothetical comets with $q<3$ AU. The distribution of OOC comets with $T<10^{9} \mathrm{yr}$ has a first peak in the interval $a=(4.5-5) \cdot 10^{4} \mathrm{AU}$, and a second peak in the range $a=(1-1.5) \cdot 10^{4} \mathrm{AU}$. The combined flux of IOC and OOC comets with $T<10^{9}$ yr has a peak in the range $a=(1-1.5) \cdot 10^{4}$ AU. The distribution of semimajor axes of observed comets with $q<3$ AU has a peak in the range $(2-2.5) \cdot 10^{4} \mathrm{AU}$, and smaller peaks in the intervals $(4-4.5) \cdot 10^{4} \mathrm{AU}$ and $(1-1.5) \cdot 10^{4} \mathrm{AU}$.

The flux of OC comets with $10^{9}<T(\mathrm{yr})<2 \cdot 10^{9}$ has a first peak in the distribution of semimajor axes in the range $(2-2.5) \cdot 10^{4} \mathrm{AU}$, and second peak in the range $a=$ $(4.5-5) \cdot 10^{4} \mathrm{AU}$. Thus, our computed distribution of comets with $10^{9}<T$ (yr) $<2 \cdot 10^{9}$ agrees better with the observations than that with $T<10^{9} \mathrm{yr}$.

A possible explanation of the disagreement with the observations for $T<10^{9}$ yr is in the adopted initial distribution of perihelion distances of comets (Hills 1981), that corresponds to the distribution caused by a close stellar passage. Weissman (1993) concludes that we are not in a strong shower at present. Thus, hereafter we consider mainly results obtained after $10^{9} \mathrm{yr}$ of the evolution of our comet orbits.

\section{The flux of dynamically 'new' Oort cloud comets}

Comets with original semimajor axes $a>10^{4}$ AU entering from the Oort cloud into the planetary system for the first time are conventionally defined as 'new' comets (Oort 1950). Here are also included within the category of 'new' comets some comets with $a<10^{4}$ AU coming in the region $q<35$ AU for the first time, since comets with $a<10^{4}$ $\mathrm{AU}$ can be injected into the inner planetary system by the action stars passing within $10^{4}$ AU from the Sun.

Figure 1 shows the relative flux of 'new' comets from the outer (Fig. 1a) and the inner (Fig. 1b) parts of the Oort cloud versus perihelion distance. The flux is normalized to its value at $q<1.5 \mathrm{AU}$ with $10^{9}<\tau(\mathrm{yr})<2 \cdot 10^{9}$. The flux of 'new' comets through the outer planetary region is higher than the flux trough the inner planetary region (here defined by $q<15 \mathrm{AU})$.

In the steady-state situation, 'new' comets from the outer Oort cloud (Fig. 1a) come roughly uniformly to the perihelion distance $q<15$ AU. However, the flux of these comets in the region $15<q(\mathrm{AU})<31$ is $\sim 1.7$ times as large as the flux with $q<15 \mathrm{AU}$ (Fig. 1a). The ratio of the flux of 'new' comets from the outer Oort cloud near the orbits 


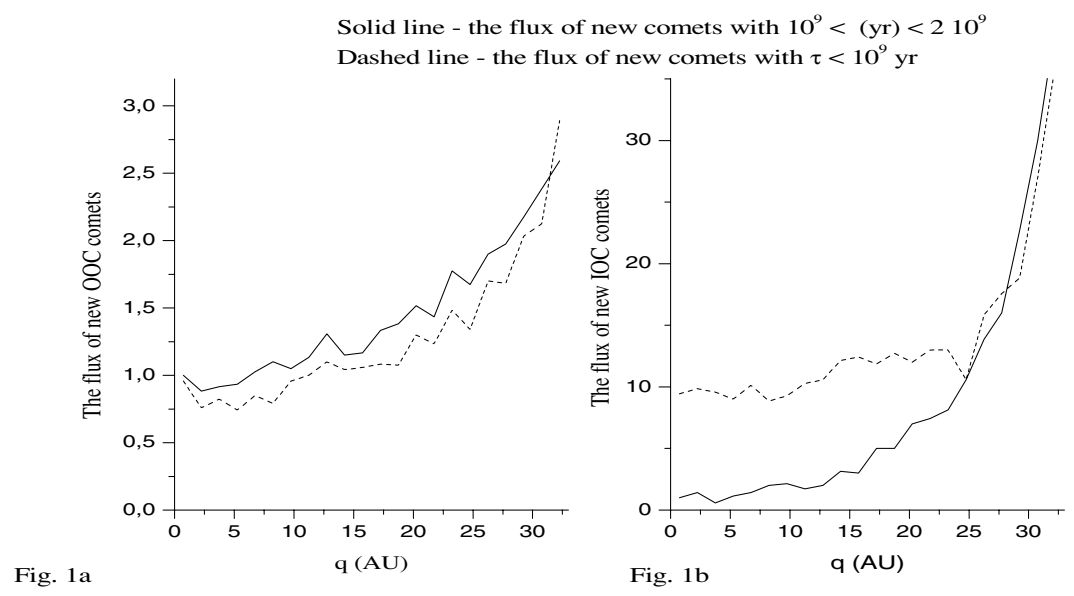

Figure 1. The relative flux of 'new' comets are coming from the outer (Fig. 1a) and inner (Fig. 1b) Oort cloud in the interval $(q, q+d q)$, where $q \leqslant 31.5 \mathrm{AU}, d q=1.5 \mathrm{AU}$. The flux is normalized to its value at $q<1.5 \mathrm{AU}$ with $10^{9}<\tau<2 \cdot 10^{9}, \tau$ is the time of perihelion passage.

of Jupiter $(5-6)$ AU, Saturn $(9-10)$ AU, Uranus $(19-20)$ AU and Neptune $(30-31)$ $\mathrm{AU}$ to the flux of these comets with $q<1 \mathrm{AU}$ is, respectively, 1, 1, 1.4 and 2.4 (Fig. 1a).

The distribution shown in Fig. 1a for $10^{9}<\tau(\mathrm{yr})<2 \cdot 10^{9}$ is slightly different from that for $\tau<10^{9}$ yr. However, the distribution of perihelion distances of 'new' comets from the inner cloud (Fig. 1b) for $10^{9}<\tau$ (yr) $<2 \cdot 10^{9}$ is very different from that for $\tau<10^{9}$ yr. Thus, the steady-state flux of comets cannot come from the inner part Oort cloud uniformly in perihelion distance for the following reasons:

- the galactic potential and stellar encounters remove comets from the inner core of the Oort cloud ( $\left.a<10^{4} \mathrm{AU}\right)$ mainly to the outside of the planetary system;

- comets of the inner core can be injected in the 'loss cone' only by the action of stars passing within $10^{4} \mathrm{AU}$ of the Sun. While the passages of stars through the hypothetical inner cloud are very rare, the passages within the outer Oort cloud are not (Heisler et al. 1987);

- within the region $q<10$ AU the planetary perturbations move comets quickly, either to hyperbolic orbits, or to orbits that are more tightly bound to the Solar system.

The ratio of the flux of 'new' comets from the inner cloud in the outside planetary region $(15<q(\mathrm{AU})<31)$ to that in the region $q<15 \mathrm{AU}$ is $\sim 7$ (Fig. 1b). The flux of comets coming from the inner Oort cloud for the first time near the orbits of Jupiter, Saturn, Uranus and Neptune to that with $q<1 \mathrm{AU}$ is 1.4, 1.6, 5.8 and 27.4 respectively (Fig. 1b).

\section{The frequency of passages of comets within the planetary region}

'New' comets may pass repeatedly through the planetary region. Comets with $a>10^{3}$ AU passing more than once within the region $q<35$ AU are defined as dynamically 'young' comets. Comets with $a<10^{3} \mathrm{AU}$, which passed many times through the planetary system, are defined as 'old' comets.

The orbital elements were printed for each perihelion passage within $q<35$ AU with $a>34.2 \mathrm{AU}$. The frequency $f(q)$ of passages of comets in the interval of perihelion distance $(q, q+d q)$ per year is calculated by

$$
f(q)=\frac{n(q)}{N \cdot t}\left(y r^{-1}\right)
$$


Table 2. The frequency of passages of dynamically new comets $\left(f_{\text {new }}\right)$, comets with $a>10^{4} \mathrm{AU}$ $\left(f_{a>10^{4}}\right)$, young comets with semimajor axes in the range $\left(10^{3} ; 10^{4}\right) \mathrm{AU}\left(f_{\text {young }}\right)$, long-period comets with $a>34.2 \mathrm{AU}\left(f_{L P}\right)$, originating from the outer $\left(f_{O O C}\right)$ and inner Oort clouds $\left(f_{I O C}\right)$ per year in the interval $(q, q+d q)$, where $q \leqslant 31.5 \mathrm{AU}, d q=1.5 \mathrm{AU}\left(10^{9} \mathrm{yr}<\tau<2 \cdot 10^{9} \mathrm{yr}\right)$.

\begin{tabular}{|c|c|c|c|c|c|c|c|c|}
\hline \multirow[t]{2}{*}{$q(\mathrm{AU})$} & \multicolumn{4}{|c|}{$f_{O O C}\left(y r^{-1}\right)$} & \multicolumn{4}{|c|}{$f_{I O C}\left(y r^{-1}\right)$} \\
\hline & $f_{\text {new }}$ & $f_{a>10^{4}}$ & $f_{\text {young }}$ & $f_{L P}$ & $f_{n e w}$ & $f_{a>10^{4}}$ & $f_{\text {young }}$ & $f_{L P}$ \\
\hline $0.0-1.5$ & $1.20 \cdot 10^{-12}$ & $1.41 \cdot 10^{-12}$ & $1.43 \cdot 10^{-12}$ & $5.28 \cdot 10^{-12}$ & $7.00 \cdot 10^{-14}$ & $6.00 \cdot 10^{-14}$ & $1.70 \cdot 10^{-13}$ & $3.50 \cdot 10^{-13}$ \\
\hline $1.5-3.0$ & $1.06 \cdot 10^{-12}$ & $1.50 \cdot 10^{-12}$ & $2.47 \cdot 10^{-12}$ & $8.98 \cdot 10^{-11}$ & $1.00 \cdot 10^{-13}$ & $1.00 \cdot 10^{-13}$ & $3.80 \cdot 10^{-13}$ & $1.46 \cdot 10^{-12}$ \\
\hline $3.0-4.5$ & $1.10 \cdot 10^{-12}$ & $1.69 \cdot 10^{-12}$ & $5.14 \cdot 10^{-12}$ & $3.56 \cdot 10^{-11}$ & $4.00 \cdot 10^{-14}$ & $1.10 \cdot 10^{-13}$ & $7.70 \cdot 10^{-13}$ & $4.17 \cdot 10^{-12}$ \\
\hline $4.5-6.0$ & $1.12 \cdot 10^{-12}$ & $1.84 \cdot 10^{-12}$ & $9.60 \cdot 10^{-12}$ & $8.80 \cdot 10^{-11}$ & $8.00 \cdot 10^{-14}$ & $1.60 \cdot 10^{-13}$ & $3.18 \cdot 10^{-12}$ & $2.11 \cdot 10^{-11}$ \\
\hline $6.0-7.5$ & $1.23 \cdot 10^{-12}$ & $2.66 \cdot 10^{-12}$ & $2.54 \cdot 10^{-11}$ & $1.58 \cdot 10^{-10}$ & $1.00 \cdot 10^{-13}$ & $2.60 \cdot 10^{-13}$ & $6.90 \cdot 10^{-12}$ & $7.38 \cdot 10^{-11}$ \\
\hline $7.5-9.0$ & $1.32 \cdot 10^{-12}$ & $4.08 \cdot 10^{-12}$ & $3.25 \cdot 10^{-11}$ & $4.01 \cdot 10^{-10}$ & $1.40 \cdot 10^{-13}$ & $4.90 \cdot 10^{-13}$ & $3.08 \cdot 10^{-11}$ & $4.17 \cdot 10^{-10}$ \\
\hline $9.0-10.5$ & $1.26 \cdot 10^{-12}$ & $4.63 \cdot 10^{-12}$ & $1.98 \cdot 10^{-11}$ & $1.04 \cdot 10^{-9}$ & $1.50 \cdot 10^{-13}$ & $8.40 \cdot 10^{-13}$ & $7.71 \cdot 10^{-11}$ & $7.60 \cdot 10^{-10}$ \\
\hline $10.5-12.0$ & $1.36 \cdot 10^{-12}$ & $5.78 \cdot 10^{-12}$ & $6.84 \cdot 10^{-11}$ & $9.04 \cdot 10^{-10}$ & $1.20 \cdot 10^{-13}$ & $1.37 \cdot 10^{-12}$ & $7.76 \cdot 10^{-11}$ & $3.96 \cdot 10^{-9}$ \\
\hline $12.0-13.5$ & $1.57 \cdot 10^{-12}$ & $8.37 \cdot 10^{-12}$ & $5.95 \cdot 10^{-11}$ & $2.44 \cdot 10^{-10}$ & $1.40 \cdot 10^{-13}$ & $1.83 \cdot 10^{-12}$ & $1.73 \cdot 10^{-10}$ & $3.24 \cdot 10^{-9}$ \\
\hline $13.5-15.0$ & $1.38 \cdot 10^{-12}$ & $9.43 \cdot 10^{-12}$ & $1.31 \cdot 10^{-10}$ & $3.33 \cdot 10^{-10}$ & $2.20 \cdot 10^{-13}$ & $2.20 \cdot 10^{-12}$ & $1.59 \cdot 10^{-10}$ & $2.41 \cdot 10^{-9}$ \\
\hline $15.0-16.5$ & $1.40 \cdot 10^{-12}$ & $1.04 \cdot 10^{-11}$ & $1.02 \cdot 10^{-10}$ & $2.81 \cdot 10^{-10}$ & $2.10 \cdot 10^{-13}$ & $2.80 \cdot 10^{-12}$ & $1.54 \cdot 10^{-10}$ & $2.52 \cdot 10^{-9}$ \\
\hline $16.5-18.0$ & $1.60 \cdot 10^{-12}$ & $1.17 \cdot 10^{-11}$ & $6.65 \cdot 10^{-11}$ & $5.34 \cdot 10^{-10}$ & $3.50 \cdot 10^{-13}$ & $3.04 \cdot 10^{-12}$ & $1.49 \cdot 10^{-10}$ & $2.18 \cdot 10^{-9}$ \\
\hline $18.0-19.5$ & $1.66 \cdot 10^{-12}$ & $1.25 \cdot 10^{-11}$ & $7.98 \cdot 10^{-11}$ & $2.60 \cdot 10^{-10}$ & $3.50 \cdot 10^{-13}$ & $3.08 \cdot 10^{-12}$ & $0^{-10}$ & $2.05 \cdot 10^{-9}$ \\
\hline $19.5-21.0$ & $1.82 \cdot 10^{-12}$ & $1.36 \cdot 10^{-11}$ & $9.36 \cdot 10^{-11}$ & $2.16 \cdot 10^{-10}$ & $4.90 \cdot 10^{-13}$ & $3.08 \cdot 10^{-12}$ & $2.72 \cdot 10^{-10}$ & $2.69 \cdot 10^{-9}$ \\
\hline $21.0-22.5$ & $1.72 \cdot 10^{-12}$ & $1.39 \cdot 10^{-11}$ & $5.92 \cdot 10^{-11}$ & $1.80 \cdot 10^{-10}$ & $5.20 \cdot 10^{-13}$ & $3.37 \cdot 10^{-12}$ & $4.44 \cdot 10^{-10}$ & $2.82 \cdot 10^{-9}$ \\
\hline $22.5-24.0$ & $2.13 \cdot 10^{-12}$ & $1.61 \cdot 10^{-11}$ & $7.01 \cdot 10^{-11}$ & $6.45 \cdot 10^{-10}$ & $5.70 \cdot 10^{-13}$ & $3.54 \cdot 10^{-12}$ & $4.88 \cdot 10^{-10}$ & $3.04 \cdot 10^{-9}$ \\
\hline $24.0-25.5$ & $2.01 \cdot 10^{-12}$ & $1.68 \cdot 10^{-11}$ & $6.88 \cdot 10^{-11}$ & $4.46 \cdot 10^{-10}$ & $7.40 \cdot 10^{-13}$ & $3.71 \cdot 10^{-12}$ & $4.83 \cdot 10^{-10}$ & $3.63 \cdot 10^{-9}$ \\
\hline $25.5-27.0$ & $2.28 \cdot 10^{-12}$ & $1.85 \cdot 10^{-11}$ & $5.32 \cdot 10^{-11}$ & $1.99 \cdot 10^{-10}$ & $9.70 \cdot 10^{-13}$ & $3.73 \cdot 10^{-12}$ & $3.85 \cdot 10^{-10}$ & $5.40 \cdot 10^{-9}$ \\
\hline $27.0-28.5$ & $2.37 \cdot 10^{-12}$ & $1.87 \cdot 10^{-11}$ & $9.15 \cdot 10^{-11}$ & $1.62 \cdot 10^{-10}$ & $1.12 \cdot 10^{-12}$ & $4.17 \cdot 10^{-12}$ & $5.62 \cdot 10^{-10}$ & $4.63 \cdot 10^{-9}$ \\
\hline $28.5-30.0$ & $2.61 \cdot 10^{-12}$ & $2.06 \cdot 10^{-11}$ & $7.22 \cdot 10^{-11}$ & $9.73 \cdot 10^{-11}$ & $1.59 \cdot 10^{-12}$ & $4.48 \cdot 10^{-12}$ & $3.78 \cdot 10^{-10}$ & $3.51 \cdot 10^{-9}$ \\
\hline $30.0-31.5$ & $2.86 \cdot 10^{-12}$ & $1.99 \cdot 10^{-11}$ & $8.98 \cdot 10^{-11}$ & $1.10 \cdot 10^{-10}$ & $2.10 \cdot 10^{-12}$ & $4.26 \cdot 10^{-12}$ & $3.15 \cdot 10^{-10}$ & $2.34 \cdot 10^{-9}$ \\
\hline $31.5-33.0$ & $3.11 \cdot 10^{-12}$ & $2.12 \cdot 10^{-11}$ & $3.34 \cdot 10^{-11}$ & $5.47 \cdot 10^{-11}$ & $2.76 \cdot 10^{-12}$ & $4.63 \cdot 10^{-12}$ & $4.39 \cdot 10^{-10}$ & $9.63 \cdot 10^{-10}$ \\
\hline
\end{tabular}

where $n(q)$ is number of perihelion passages of comets in a given interval $(q, q+d q), N$ is the population of hypothetical comets in the inner or outer Oort cloud $\left(N=10^{5}\right)$, $t$ is the investigated time interval $\left(t=10^{9} \mathrm{yr}\right)$. Table 2 shows the computed frequency $f(q)$ of passages of dynamically new comets $\left(f_{\text {new }}\right)$, new and young comets with a $>10^{4}$ $\mathrm{AU}\left(f_{a>10^{4}}\right)$, young comets with semimajor axes in the range $\left(10^{3} ; 10^{4}\right) \mathrm{AU}\left(f_{\text {young }}\right)$, long-period comets with $a>34.2 \mathrm{AU}\left(f_{L P}\right)$, originating from the outer $\left(f_{O O C}\right)$ and inner cloud $\left(f_{I O C}\right)$, in the interval $(q, q+d q)$, where $q<31.5 \mathrm{AU}, d q=1.5 \mathrm{AU}$.

Bailey \& Stagg (1988) estimated that one comet with $a>10^{4} \mathrm{AU}$ and $H_{10}<7$ (diameter in the range $5-15 \mathrm{~km}$ ) passes perihelion with $q<1$ AU every 5 years, while Fernández (2002) estimated this frequency at every 3 years. Let $n(1)$ be the number of comets coming in the region $q<1 \mathrm{AU}$ per year with $a>10^{4} \mathrm{AU}$; then, the population of the Oort cloud may be computed by

$$
N_{O C}=\frac{n(1)}{f_{a>10^{4}}(1) \cdot t},
$$

where $f_{a>10^{4}}(1)$ is the frequency of passages of Oort cloud comets with $a>10^{4} \mathrm{AU}$ within $q<1 \mathrm{AU}$, and $t$ is one year. The frequency $f_{a>10^{4}}(1)=4.8 \cdot 10^{-13} \mathrm{yr}^{-1}$ has been obtained from (5.1), $n(1)=0.2-0.3$ comets per year (Bailey \& Stagg 1988; Fernández 2002). The corresponding number of comets in the Oort cloud, including the inner and outer parts, with $H_{10}<7$ is equal to $N_{O C}=N_{O O C}+N_{I O C} \approx(4.2-6.3) \cdot 10^{11}$. The average number is $N_{O C} \approx 5.25 \cdot 10^{11}$ comets with $H_{10}<7$.

The number $n(1)$ should be 12 times larger for comets with $H_{10}<10.9$ according to the $10^{\alpha H_{10}}$ dependence of the cumulative distribution of the number of objects brighter than $H_{10}$ (Levison et al. 2002). From this follows that $n(1)=2.4-3.6$ comets with $H_{10}<10.9$. Then, the population of the Oort cloud is $N_{O C}=N_{O O C}+N_{I O C} \approx(5-7.5) \cdot 10^{12}$ comets 


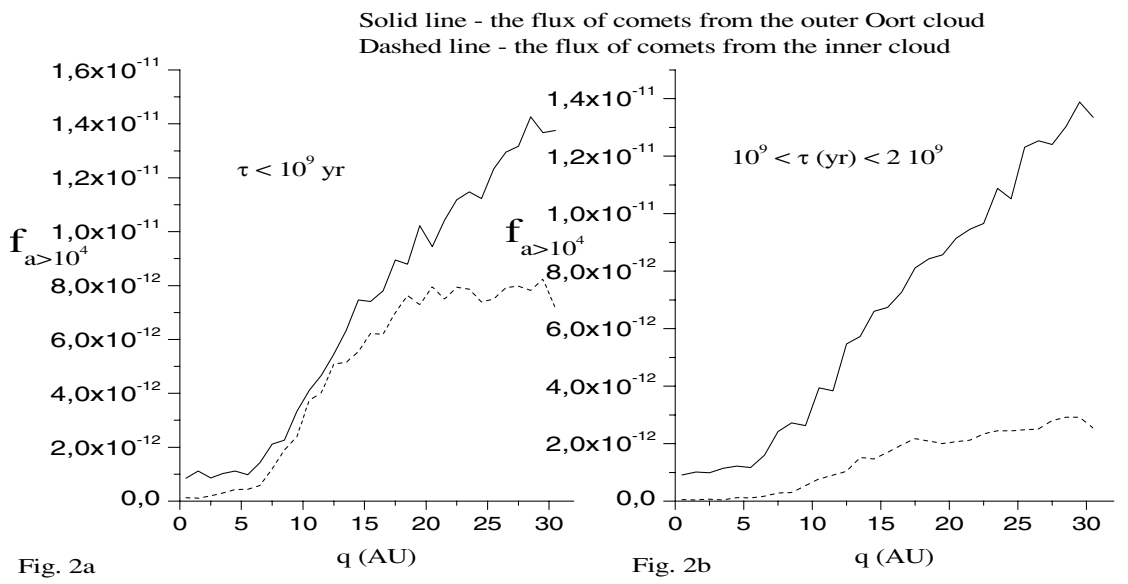

Figure 2. The frequency $f_{a>10^{4}}(q)$ of passages of comets with $a>10^{4}$ AU per year in the interval of perihelion distance $(q, q+d q)$, where $q \leqslant 30 \mathrm{AU}, d q=1 \mathrm{AU}$, for $\tau<10^{9}$ yr (Fig. 2a) and $10^{9}<\tau$ (yr) $<2 \cdot 10^{9}$ (Fig. $2 \mathrm{~b}$ ), where $\tau$ is the time of perihelion passage.

with $H_{10}<10.9$. The average number $N_{O C} \approx 6.25 \cdot 10^{12}$ comets with $H_{10}<10.9$, that is consistent with Weissman (1996). Francis (2005) found that the flux of comets through the inner solar system and the outer Oort cloud population are much lower than previously published estimates (Bailey \& Stagg 1988; Weissman 1996).

Duncan et al. (1987) studied the formation of the Oort cloud and its subsequent evolution over a time span of $4.5 \cdot 10^{9} \mathrm{yr}$, and found that approximately $70 \%$ of Oort cloud comets lie in its inner part $\left(a<10^{4} \mathrm{AU}\right)$. According to them, the inner comet cloud $\left(a<10^{4} \mathrm{AU}\right)$ acts as a source, replenishing the outer cloud $\left(a>10^{4} \mathrm{AU}\right)$. Comets evolve from the inner into the outer Oort cloud, and can be further brought into the visible region with $a>10^{4}$ AU. Thus, comets with $a>10^{4}$ AU observed in near-Earth space can originate from either the outer or the inner clouds. The calculated frequency (5.1) of passages of comets with $a>10^{4}$ AU from the outer Oort cloud within $q<1 \mathrm{AU}$ is $9.1 \cdot 10^{-13} \mathrm{yr}^{-1}$, that is roughly one order of magnitude larger than that from the inner cloud $\left(5 \cdot 10^{-14} \mathrm{yr}^{-1}\right)$. Nevertheless, the flux of comets with $a>10^{4} \mathrm{AU}$ in the region $q<1 \mathrm{AU}$ (as in the region $q<35 \mathrm{AU}$ ) comprises both outer cloud comets and inner cloud comets that have replenished the OOC. The combined flux of comets per interval $(q, q+d q)$, can be calculated by

$$
n(q)=n_{O O C}(q)+n_{I O C}(q)=N_{O O C} \cdot f_{O O C}(q)+N_{I O C} \cdot f_{I O C}(q),
$$

where $n_{O O C}(q)$ is the flux of comets from the outer Oort cloud, $f_{O O C}(q)$ is the frequency of passages of OOC comets in a given interval $(q, q+d q), N_{O O C}$ is the population of comets in the outer cloud, $N_{O O C}=N_{O C} /(k+1)$. Analogously, $n_{I O C}(q)$ is the flux of comets from the inner cloud, $f_{I O C}(q)$ is the frequency of passages of IOC comets in a given interval $(q, q+d q), N_{I O C}$ is the population of comets in the IOC, $N_{I O C}=N_{O C} \cdot k /(k+1)$.

Let $n(1) \approx 3$ comets with $a>10^{4} \mathrm{AU}$ and $H_{10}<10.9$ per year, the ratio $k=1$, $N_{O C} \approx 6.25 \cdot 10^{12}$ comets; then, the contribution from the inner cloud to this flux $(q<1$ $\mathrm{AU})$ is $n_{I O C}(1)=0.156$ comets per year, i.e. about one comet every 6.5 years.

The relative population of the inner cloud $\left(N_{I O C} / N_{O O C}=k\right)$ was determined with a large uncertainty (Hills 1981; Duncan et al. 1987; Dones et al. 2000). However, when comparing simulation results and observations, it must be considered that the flux of 
comets coming directly from the Oort cloud to near-Earth space is the less uncertain parameter. Using the ratio $N_{I O C} / N_{O O C}=k$, Eq. (5.3) can be written as:

$$
n(q)=N_{O O C} \cdot\left(f_{O O C}(q)+f_{I O C}(q) \cdot k\right) .
$$

From (5.4), using the model data (Table 2), one can compute the flux of comets in every interval $(q, q+d q)$, where $q<31.5 \mathrm{AU}, d q=1.5 \mathrm{AU}$, depending on value of $k$.

Figure 2 shows the distributions $f_{a>10^{4}}(q)$ for $\tau<10^{9}$ yr and $10^{9}<\tau(\mathrm{yr})<2 \cdot 10^{9}$, where $f_{a>10^{4}}(q)$ is the frequency of passages of comets with $a>10^{4}$ AU per year in the interval of perihelion distance $(q, q+d q), q \leqslant 30 \mathrm{AU}, d q=1$ AU. The maxima in these distributions $f_{a>10^{4}}(q)$ are located in the outer part of the planetary region, near the orbit of Neptune (Fig. 2). Comets with $a>10^{4}$ AU mainly come from the outer cloud.

Fernández (2002) pointed out that the flux of comets with $a>1.5 \cdot 10^{4}$ AU increases with increasing perihelion distance. The flux of outer Oort cloud comets with $a>10^{4}$ AU near the orbits of Jupiter, Saturn, Uranus and Neptune is 1.28, 2.89, 9.42, and 14.7 times larger than that with $q<1 \mathrm{AU}$. The corresponding ratios for the flux of comets with $a>10^{4}$ AU originating from the inner cloud are 2.2, 10.8, 40 and 50.8; however the frequency of passages of these comets within the planetary region is much less than the frequency of passages of OOC comets with $a>10^{4} \mathrm{AU}$ (Fig. 2).

\section{The ratio between comets with $\mathrm{a}>10^{4}$ AU and 'new' comets}

Dybczynski (2001) proposed that only 50\% comets, defined as 'new', are actually in their first revolution through the inner parts of the Solar system $(q<15 \mathrm{AU})$. However, he modified the Oort definition of dynamically 'new' comets, having changed the threshold value of inverse semimajor axis from $10^{-4} \mathrm{AU}^{-1}$ to $2.5 \cdot 10^{-4} \mathrm{AU}^{-1}$. As mentioned above, in this paper are denoted as 'new' all the comets that make their first passage in the region $q<35 \mathrm{AU}$.

Figure 3 shows the ratio $\nu(q)$ between $\nu_{a>10^{4}}(q)$ and $\nu_{\text {new }}(q)$, where $\nu_{a>10^{4}}(q)$ is the number of perihelion passages of comets with $a>10^{4}$ AU into the region $q<31 \mathrm{AU}$, $\nu_{\text {new }}(q)$ is the number of perihelion passages of 'new' comets.

\subsection{The ratio $\nu(q)$ for comets from the outer Oort cloud}

The computed frequency of passages of 'new' comets $\left(f_{\text {new }}\right)$ from the outer Oort cloud is equal to $1.2 \cdot 10^{-12} \mathrm{yr}^{-1}$ within $q<1.5 \mathrm{AU}$ (Table 2 ). The frequency of passages of outer cloud comets with $a>10^{4} \mathrm{AU}$ within the region $q<1.5 \mathrm{AU}$ is $f_{a>10^{4}}(1.5)=1.41 \cdot 10^{-12}$ $\mathrm{yr}^{-1}$ (Table 2). The ratio $\nu(q)$ for OOC comets is equal to 1.17 in the region $q<1.5 \mathrm{AU}$.

The number of returns in the planetary region of comets with $a>10^{4} \mathrm{AU}$ depends on $q$ (Fig. 3). For small $q$ planetary perturbations are strong enough to remove most of comets from the outer Oort cloud, either to hyperbolic orbits or to smaller elliptical orbits. Outside the orbit of Jupiter, the number of comets with $a>10^{4}$ AU returning to the planetary region increases rapidly with increasing $q$ (Fig. 3). Our computations show that in the region $q<15$ AU approximately $30 \%$ of comets from the outer Oort cloud with $a>10^{4}$ AU are really on their first passage in the planetary system.

\subsection{The ratio $\nu(q)$ for comets from the inner cloud}

Figure 3 shows also the ratio $\nu(q)$ for comets originating from the inner cloud (dashed line). The obtained frequency of passages of new comets $\left(f_{\text {new }}\right)$ from the inner Oort cloud within $q<1.5 \mathrm{AU}$ is equal to $7 \cdot 10^{-14} \mathrm{yr}^{-1}$ (Table 2) that is more than one order of 


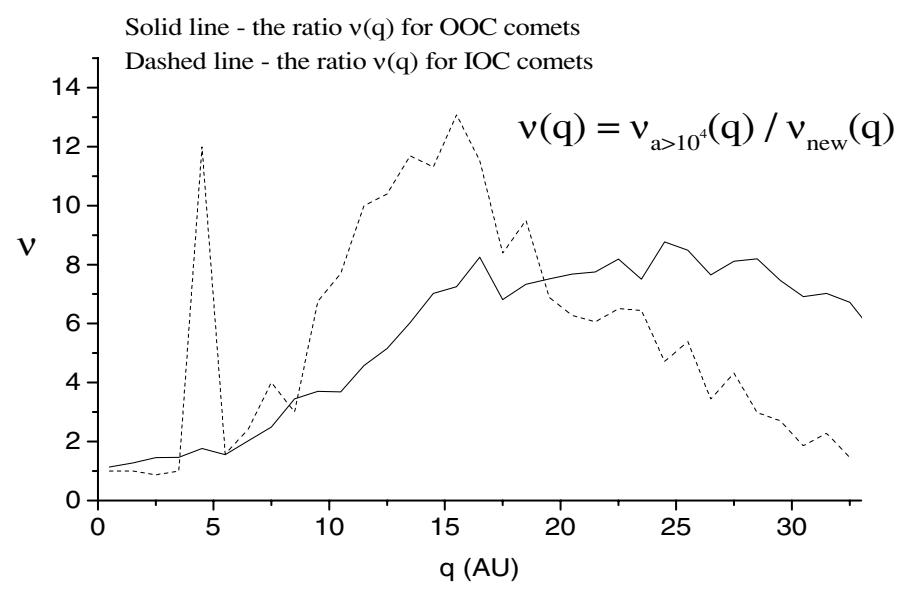

Figure 3. The ratio $\nu=\nu_{a>10^{4}} / \nu_{\text {new }}$ as a function of $q$, where $\nu_{a>10^{4}}$ is the number of perihelion passages of comets with $a>10^{4} \mathrm{AU}, \nu_{\text {new }}$ is number of perihelion passages of 'new' comets.

magnitude less than the frequency of passages of new comets from the outer cloud. The ratio $\nu(q)$ has peaks near the orbit of Jupiter and in the Saturn-Uranus region (Fig. 3).

Comets from the inner cloud $\left(a<10^{4} \mathrm{AU}\right)$ that come to the region $q<35$ AU may be removed to orbits with $a>10^{4}$ AU by the planetary perturbations, and become subject by the stronger external perturbations. In fact, galactic and stellar perturbations can change the perihelion distances of these comets, bringing the perihelia of some of them into the visible region. The majority of outer Oort cloud comets evolve rapidly into smaller orbits, before their possible deflection by the action of giant planets back into the Oort region. Comets of the inner cloud evolve very slowly in the outside planetary region, and would be removed to orbits with $a>10^{4}$ AU more likely than dynamically young or old comets originating from the OOC. The majority of IOC comets deflected due to the planetary perturbations into the outer cloud $\left(a>10^{4} \mathrm{AU}\right)$ come from orbits with semimajor axes in the range $5 \cdot 10^{3}<a(\mathrm{AU})<10^{4}$.

Our computations show that in the region $q<15$ AU only $5 \%$ of comets with $a>10^{4}$ AU originating from the inner Oort cloud are on their first passage in the planetary system. Thus the majority of IOC comets move to orbits with $a>10^{4}$ AU within the planetary region, mainly in the Saturn-Uranus region (Fig. 3).

\section{Differences in the dynamical evolution of young comets from the inner and outer Oort cloud in the planetary region}

Figure 4 shows the distributions $f_{\text {young }}(q)$ for $\tau<10^{9}$ yr (Fig. 4a) and $10^{9}<\tau$ yr $<2 \cdot 10^{9}$ (Fig. $4 \mathrm{~b}$ ), where $f_{\text {young }}(q)$ is the frequency of passages of dynamically young comets with semimajor axes in the range $10^{3}<a(\mathrm{AU})<10^{4}$ per year in the interval of perihelion distance $(q, q+d q), q \leqslant 30 \mathrm{AU}, d q=1 \mathrm{AU}$.

\subsection{The dynamical evolution of young comets from the outer Oort cloud}

In the Uranus-Neptune region $(q \approx 20-30$ AU) the evolution of cometary orbits under planetary perturbations is very slow, consequently galactic and stellar perturbations are important in this region. The perihelia of 'new' comets coming from the outer Oort cloud with $a>(1.5-2) \cdot 10^{4} \mathrm{AU}$ in the region $q>20 \mathrm{AU}$ are quickly transferred to 


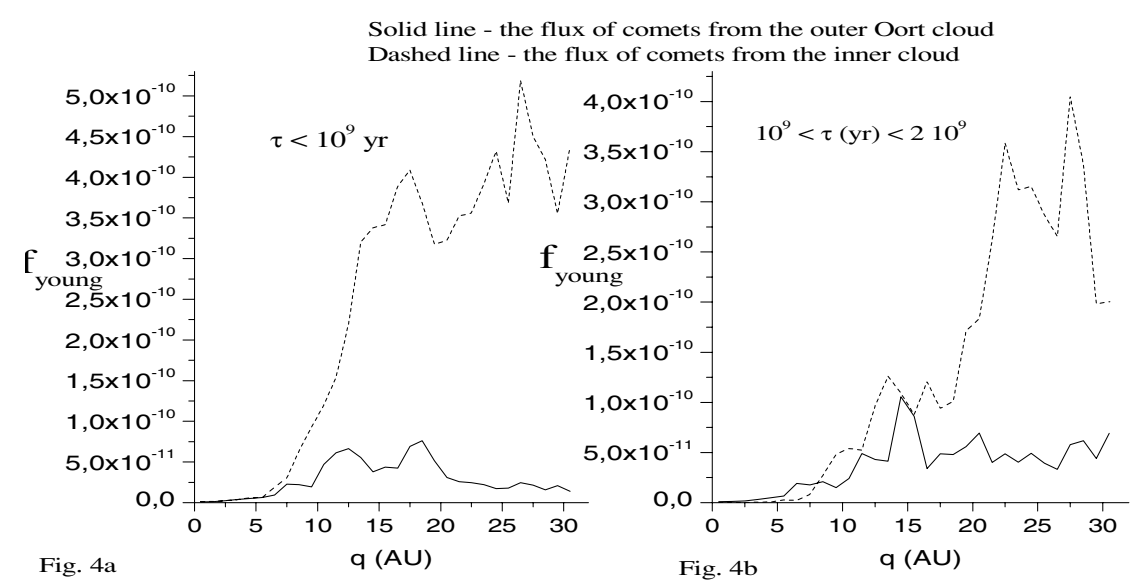

Figure 4. The frequency $f_{\text {young }}(q)$ of passages of comets with semimajor axes in the range $10^{3}<a(\mathrm{AU})<10^{4}$ per year in the interval of perihelion distance $(q, q+d q)$, where $q \leqslant 30$ $\mathrm{AU}, d q=1 \mathrm{AU}$, for $\tau<10^{9}$ yr (Fig. 4a) and $10^{9}<\tau$ (yr) $<2 \cdot 10^{9}$ (Fig. 4b), $\tau$ is the time of perihelion passage.

$q<20$ AU by the action of external perturbations. In a passage through the 'loss cone' $(q<10 \mathrm{AU})$ a comet undergoes a change in its reciprocal semimajor axes $\Delta \varepsilon$ of about $\Delta \varepsilon \approx\left(10^{-4}-10^{-3}\right) \mathrm{AU}^{-1}$ (Fernández 1981), and is rapidly removed from the Oort cloud, either to smaller elliptical orbits or to hyperbolic orbits. In the region $q \approx(10-20) \mathrm{AU}$ planetary perturbations are weaker, $\Delta \varepsilon \approx\left(10^{-5}-10^{-4}\right) \mathrm{AU}^{-1}$, therefore in this region the number of dynamically young comets returning to the planetary region increases. Thus, most of the perihelia of dynamically young comets with $a>10^{3}$ AU from the outer Oort cloud are located in the Saturn-Jupiter region.

\subsection{The dynamical evolution of young comets from the inner cloud}

The maximum in the perihelion distribution of inner cloud comets on their first passage through the planetary region lies near Neptune's orbit, therefore IOC comets mainly come in the outer planetary region from the nearest regions $(q>35 \mathrm{AU})$. In the UranusNeptune region planetary perturbations are too weak $\left(\Delta \varepsilon \leqslant 10^{-5} \mathrm{AU}^{-1}\right.$ per perihelion passage) to remove comets with $a<10^{4} \mathrm{AU}$. Comets from the inner cloud will drift in $q$ very smoothly under the action of galactic perturbations in the Uranus-Neptune region.

Comets coming into the Jupiter-Saturn region will be affected by stronger planetary perturbations, and evolve into smaller orbits more rapidly. Therefore the flux of dynamically young comets from the inner cloud to the outer planetary region $q>20$ AU is the largest (Fig. 4b).

Thus, the flux of dynamically young comets from the inner cloud is largest in the Uranus-Neptune region (Fig. 4), while the flux of dynamically young comets from the outer Oort cloud is largest in the Saturn-Uranus region (Fig. 4).

\section{Oort cloud comets near the orbit of the Earth}

'New' comets come directly from the Oort cloud into the inner solar system, and also in the near-Earth space $(q<1.5 \mathrm{AU})$. Those Oort cloud comets that come in the outer planetary region can evolve gradually to small perihelion distances. Thus there are two dynamical ways by which Oort cloud comets can reach near-Earth space. 
The frequency of injection of outer Oort cloud comets directly from $q>35$ AU to $q<1.5 \mathrm{AU}$ is $1.2 \cdot 10^{-12} \mathrm{yr}^{-1}$ (Table 2). This is the frequency of passages of 'new' comets. The frequency of supply of OOC comets evolving gradually to $q<1.5$ AU from the outer planetary region is $5.6 \cdot 10^{-13} \mathrm{yr}^{-1}$. The frequency of injection of inner cloud comets directly from $q>35$ AU into the region $q<1.5 \mathrm{AU}$ is $7 \cdot 10^{-14} \mathrm{yr}^{-1}$ (Table 2). Comets from the inner cloud can be injected from $q>35$ AU directly to $q<1.5 \mathrm{AU}$ only during comet showers due to a very close star passage (Hills 1981). From the outside planetary region IOC comets come gradually to $q<1.5 \mathrm{AU}$ with frequency $1.5 \cdot 10^{-13}$ $\mathrm{yr}^{-1}$. Thus, the majority of IOC comets reach the region $q<1.5$ AU gradually evolving from the outer planetary region, while the majority of OOC comets come to $q<1.5 \mathrm{AU}$ directly from $q>35 \mathrm{AU}$.

Considering comets that are coming gradually into the region $q<1.5$ AU, let us examine their orbital elements on their previous perihelion passage, before entering to $q<1.5 \mathrm{AU}$ region. The maximum in the distribution of perihelion distances of comets on their previous passage lies near the orbit of Saturn. The majority of the semimajor axes of IOC comets were located in the range $(0.5-1.5) \cdot 10^{4} \mathrm{AU}$. The semimajor axes of OOC comets before their entry to $q<1.5$ AU were located mainly in the range $(0.5-3) \cdot 10^{4}$ AU, with the maximum in the interval $(2.5-3) \cdot 10^{4}$ AU. Therefore, dynamically young comets with semimajor axes of a few thousand AU $\left(a<10^{4} \mathrm{AU}\right)$ can also be perturbed from orbits with perihelia beyond the orbits of Saturn or Jupiter to perihelia inside the orbit of Jupiter. Near the orbit of Saturn these comets can be removed to orbits with $a>10^{4} \mathrm{AU}$, become subject to strong external perturbations, and then come to the visible region on their next passage. The majority of new comets come into the outer planetary region. A fraction of $\sim 0.015$ new OOC comets and a fraction of $\sim 0.011$ new IOC comets can reach orbits with $q<1.5$ AU from $15<q<35$ AU. The majority of the perihelia of IOC comets reaching near-Earth space $(q<1.5 \mathrm{AU})$, on their first passage in the planetary region were located in the interval $q=(30-35)$ AU.

The frequency of passages of long-period $(a>34.2 \mathrm{AU})$ comets from the inner cloud within $q<1.5 \mathrm{AU}$ is $3.5 \cdot 10^{-13} \mathrm{yr}^{-1}$, that is roughly one order of magnitude less than the frequency of passages of LP comets from the outer Oort cloud $\left(5.28 \cdot 10^{-12} \mathrm{yr}^{-1}\right)$. As mentioned above, the frequency of passages of new comets from the OOC within $q<1.5$ AU is $1.2 \cdot 10^{-12} \mathrm{yr}^{-1}$ (Table 2), and from the IOC is $7 \cdot 10^{-14} \mathrm{yr}^{-1}$. Thus the ratio of LP comets with $q<1.5$ AU to new comets is $\sim 5$. Bailey \& Stagg (1988) estimated that the flux of long-period comets with $H_{10}<7$ near the Earth $(q<1 \mathrm{AU})$ is $1.0 \pm 0.2 \mathrm{yr}^{-1}$ $\mathrm{AU}^{-1}$. Let us assume that the ratio $k=1$, the Oort cloud contains $5.25 \cdot 10^{11}$ comets with $H_{10}<7$ or $6.25 \cdot 10^{12}$ comets with $H_{10}<10.9$, then from (5.4) the flux of long-period comets within $q<1$ AU is equal to $0.8 \mathrm{yr}^{-1}\left(H_{10}<7\right)$, that is consistent with Bailey \& Stagg (1988). Therefore, we can estimate the flux of comets in the vicinity of the Earth.

The contribution of OOC comets to the near-Earth flux $(q<1.5 \mathrm{AU})$ with $H_{10}<7$ is $\sim 1.4 \mathrm{LP}$ comets per year, the contribution of IOC comets to this flux is $\sim 0.1 \mathrm{LP}$ comets per year. Thus, the total flux of LP comets from the Oort cloud with $q<1.5$ AU is approximately 1.5 comets per year $\left(H_{10}<7\right)$. The total flux of LP comets within $q<1.5 \mathrm{AU}$ with $H_{10}<10.9$ is $\sim 18$ comets per year, of them $\sim 1.1 \mathrm{LP}$ comets originate from the inner cloud. The flux of new comets with $H_{10}<7$ to $q<1.5$ AU is $\sim 0.34$ comets per year, of them $\sim 0.02$ new comets come from the inner cloud (on average one comet per 50 years). The flux of new comets to $q<1.5$ AU with $H_{10}<10.9$ is $\sim 4$ comets per year, of them $\sim 0.22$ new comets from the inner cloud (on average one comet per 4.5 years). The frequency of passages of IOC comets that have been transferred to the OOC with $a>10^{4} \mathrm{AU}$ within $q<1.5 \mathrm{AU}$ is $6 \cdot 10^{-14} \mathrm{yr}^{-1}$, that is more than one order of magnitude less than the frequency of passages of outer cloud comets with $a>10^{4} \mathrm{AU}$ 


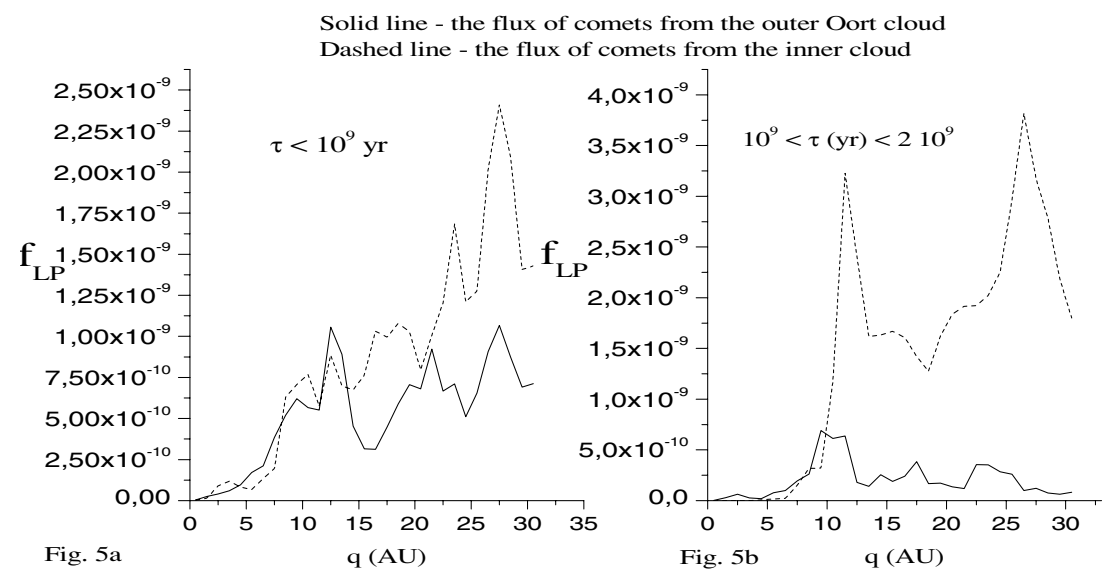

Figure 5. The frequency $f_{L P}(q)$ of passages of LP comets $(a>34.2 \mathrm{AU})$ per year in the interval of perihelion distance $(q, q+d q)$, where $q \leqslant 30 \mathrm{AU}, d q=1 \mathrm{AU}$, for $\tau<10^{9} \mathrm{yr}$ (Fig. $5 \mathrm{a}$ ) and $10^{9}<\tau$ (yr) $<2 \cdot 10^{9}$ (Fig. $5 \mathrm{~b}$ ), $\tau$ is the time of perihelion passage.

$\left(1.41 \cdot 10^{-12} \mathrm{yr}^{-1}\right)$. The flux of OC comets with $a>10^{4} \mathrm{AU}$ within $q<1.5 \mathrm{AU}$ is 4.6 comets with $H_{10}<10.9$ per year, and is 0.4 comets per year with $H_{10}<7$.

\section{Dynamically old comets}

Figure 5 shows the distributions $f_{L P}(q)$ for $\tau<10^{9}$ yr (Fig. 5a) and $10^{9}<\tau$ (yr) $<2 \cdot 10^{9}$ (Fig. 5b), where $f_{L P}(q)$ is the frequency of passages of long-period (LP) comets with semimajor axes $a>34.2$ AU per year in the interval of perihelion distance $(q, q+d q)$, $q \leqslant 30 \mathrm{AU}, d q=1 \mathrm{AU}$.

The flux of comets with $a>34.2$ AU originating from the inner Oort cloud strongly predominates in the region $q>10 \mathrm{AU}$ (Fig. 5). The orbits of IOC comets evolve slowly to the outer planetary region. The flux of LP comets originating from the OOC is predominant within $q<10 \mathrm{AU}$. Comets of the outer cloud evolve into smaller orbits more rapidly than comets of the inner cloud. Inner cloud comets enter the planetary region with much larger perihelion distances, since they are only weakly affected by external perturbations. Comets with $a<(1.5-2) \cdot 10^{4}$ AU coming in the region $q>20 \mathrm{AU}$, besides, are weakly perturbed by the planets, and evolve slowly in the outside planetary system. Perturbations by Saturn act as a substantial barrier to the diffusion of cometary perihelia into the inner planetary system. The perihelia of comets, slowly drifting in $q$, will accumulate beyond the orbit of Saturn. The distributions of the frequency $f_{L P}(q)$ of passages of outer and inner cloud comets with $10^{9}<\tau(\mathrm{yr})<2 \cdot 10^{9}$ have peaks slightly beyond the orbit of Saturn (Fig. 5).

\section{Conclusions}

The dynamical evolution of outer Oort cloud comets in the planetary region is faster than that of inner cloud comets. Outer cloud comets evolve into smaller orbits more rapidly. The flux of long-period comets originating from the inner cloud is substantially predominant in the region $q>10 \mathrm{AU}$, whilethe flux of LP comets from the outer cloud is dominant in the region $q<10 \mathrm{AU}$. The flux of dynamically young and old comets originating from the outer Oort cloud is largest in the Saturn-Uranus region. The flux of dynamically young comets originating from the inner cloud is largest in the UranusNeptune region. 
Inner cloud comets $\left(a<10^{4} \mathrm{AU}\right)$ can replenish the outer Oort cloud $\left(a>10^{4} \mathrm{AU}\right)$. The frequency of passages of inner cloud comets that have replenished the outer cloud within $q<1.5 \mathrm{AU}$ is $6 \cdot 10^{-14} \mathrm{yr}^{-1}$, that is more than one order of magnitude less than the frequency of passages of outer cloud comets with $a>10^{4} \mathrm{AU}\left(1.41 \cdot 10^{-12} \mathrm{yr}^{-1}\right)$.

The majority of new comets come into the outer planetary region. The flux of new comets with $15<q<31$ AU is higher than with $q<15$ AU by a factor $\sim 1.7$ for comets from the outer cloud, and by a factor 7 for comets from the inner cloud. Inner cloud comets can reach the region $q<1.5$ AU, mainly evolving gradually from the outer planetary region, while the majority of outer cloud comets come to $q<1.5$ AU directly from $q>35 \mathrm{AU}$. The maximum in the distribution of perihelion distances of comets, that are coming gradually to near-Earth space, on their perihelion passage just before the entry into the region $q<1.5 \mathrm{AU}$, lies near the orbit of Saturn.

Near Saturn orbit, dynamically young comets with semimajor axes a few thousands astronomical units can be removed to orbits with $a>10^{4} \mathrm{AU}$, become subject of strong external perturbations, and therefore may come to the visible region on their next passage.

The maximum in the distribution of perihelion distances of comets with $a>10^{4} \mathrm{AU}$ lies near Neptune orbit. Perturbations by Saturn act as a substantial barrier to the diffusion of cometary perihelia into the inner planetary system. The perihelia of comets evolving in the outer planetary region will accumulate beyond Saturn's orbit. The distribution of perihelion distances of LP comet has a peak slightly beyond the orbit of Saturn.

The frequency of passages of long-period $(a>34.2 \mathrm{AU})$ comets from the inner cloud within $q<1.5 \mathrm{AU}$ is $3.5 \cdot 10^{-13} \mathrm{yr}^{-1}$, that is roughly one order of magnitude less than the frequency of passages of LP comets from the outer Oort cloud $\left(5.28 \cdot 10^{-12} \mathrm{yr}^{-1}\right)$. The ratio of LP comets with $q<1.5$ AU to new comets is $\sim 5$.

\section{Acknowledgements}

I thank the organizers for financial support enabling attendance at the meeting. This work also was supported by RFBR-Ural Grant 04-02-96042 and RFBR Grant 06-0216512 .

\section{References}

Bailey, M.E. \& Stagg, C.R. 1988, MNRAS 235, 1

Dones, L., Levison, H.F., Duncan, M. \& Weissman, P. 2000, BAAS 32, 1060

Duncan, M., Quinn, T. \& Tremaine, S. 1987, AJ 94, 1330

Dybczynski, P.A. 2001, Astron. Astrophys. 375, 643

Emel'yanenko, V.V. 1999, Evolution and source regions of asteroids and comets, Proc. IAU Coll. 173 , p. 339

Fernandez, J.A. 1981, Astron. Astrophys. 96, 26

Fernandez, J.A. 2002, Earth, Moon 85 Planets 89, 325

Francis, P.J. 2005, AJ 635, 1348

Heisler, J. \& Tremaine, S. 1986, Icarus 65, 13

Heisler, J., Tremaine, S. \& Alcock, C. 1987, Icarus 70, 269

Hills, J.G. 1981, $A J$ 86, 1730

Levison, H.F., Morbidelli, A., Dones, L., Jedicke, R., Wiegert, P.A. \& Bottke, W.F. 2002, Science 296,2212

Marsden, B.G., Sekanina, Z. \& Everhart, E. 1978, AJ 83, 64

Marsden, B.G. \& Williams, G.V. 2005, Catalogue of Cometary Orbits

Ogorodnikov, K.F. 1965, Dynamics of stellar systems (Oxford: Pergamon)

Oort, J.H. 1950, Bull. Astron. Inst. Neth. 11, 91

Weissman, P.R. 1993, BAAS 25, 1063

Weissman, P.R. 1996, Completing the Inventory of the Solar System, ASP Conf. Ser. 107, p. 265 